\title{
Macrophage ICAM-1 functions as a regulator of phagocytosis in LPS induced endotoxemia
}

\author{
Hanhui Zhong ${ }^{1} \cdot$ Haitao Lin $^{3} \cdot$ Qiongni Pang ${ }^{4}$ Jinling Zhuang ${ }^{2,4} \cdot$ Xiaolei Liu $^{2} \cdot$ Xiaolian Li $^{2}$. Jinghua Liu ${ }^{1}$. \\ Jing Tang ${ }^{1,2}$ (D)
}

Received: 28 October 2020 / Revised: 16 December 2020 / Accepted: 11 January 2021 / Published online: 21 January 2021

(c) The Author(s), under exclusive licence to Springer Nature Switzerland AG part of Springer Nature 2021

\begin{abstract}
Objective Intracellular adhesion molecule-1 (ICAM-1), a transmembrane glycoprotein belonging to the immunoglobulin superfamily, plays a critical role in mediating cell-cell interaction and outside-in cell signaling during the immune response. ICAM-1 is expressed on the cell surface of several cell types including endothelial cells, epithelial cells, leucocytes, fibroblasts, and neutrophils. Despite ICAM-1 has been detected on macrophage, little is known about the function and mechanism of macrophage ICAM-1.

Methods To investigate the role of lipopolysaccharide (LPS) in ICAM-1 regulation, both the protein and cell surface expression of ICAM-1 were measured. The phagocytosis of macrophage was evaluated by flow cytometry and Confocal microscopy. Small interfering RNA and neutralizing antibody of ICAM-1 were used to assess the effect of ICAM-1 on macrophage phagocytosis. TLR4 gene knockout mouse and cytoplasmic and mitochondrial ROS scavenger were used for the regulation of ICAM-1 expression. ROS was determined using flow cytometry.

Results In this study, we reported that macrophage can be stimulated to increase both the protein and cell surface expression of ICAM-1 by LPS. Macrophage ICAM-1 expression was correlated with enhanced macrophage phagocytosis. We found that using ICAM-1 neutralizing antibody or ICAM-1 silencing to attenuate the function or expression of ICAM-1 could decrease LPS-induced macrophage phagocytosis. Furthermore, we found that knocking out of TLR4 led to inhibited cytoplasmic and mitochondrial ROS production, which in turn, attenuated ICAM-1 expression at both the protein and cell surface levels. Conclusion This study demonstrates that the mechanism of ICAM-1-mediated macrophage phagocytosis is depending on TLR4-mediated ROS production and provides significant light on macrophage ICAM-1 in endotoxemia.
\end{abstract}

Keywords Macrophage $\cdot$ ICAM-1 $\cdot$ TLR4 $\cdot$ ROS $\cdot$ Phagocytosis

Communicated by John Di Battista.

Jinghua Liu

liujhua@smu.edu.cn

$\triangle$ Jing Tang

tanglitangjing@126.com

1 Guangdong Provincial Key Laboratory of Proteomics, School of Basic Medical Sciences, Southern Medical University, Guangzhou 510515, China

2 The Department of Anesthesiology, Affiliated Hospital of Guangdong Medical University, Zhanjiang 524000, Guangdong, China

3 Health Management Center, Affiliated Hospital of Guangdong Medical University, Zhanjiang 524000, Guangdong, China

4 The Department of Anesthesiology, Nanfang Hospital, SouthernMedicalUniversity, Guangzhou 510515, Guangdong, China

\section{Introduction}

Sepsis or endotoxemia, one of the leading cause of death in the intensive care unit [1,2], is a life-threatening condition that is caused by dysregulated the host response to infection [3]. In endotoxemia, microbes disturb host immune response and fail to regulate the inflammatory response [2, 3]. Macrophages, a first line to defend against pathogens, play a pivotal role in sepsis or endotoxemia because of their functions, such as phagocytosis, inflammatory response, chemotaxis, antigen presentation [4-6]. Phagocytosis is a complex cellular process. It requires a coordinated interaction between macrophage cell surface receptors and ligands on the surface of the particles, which leads to the dynamic rearrangement of the cytoskeleton $[7,8]$. In fact, macrophage phagocytosis depends on a variety of participants, including receptors 
(such as Fc gamma receptors, complement receptor 3, and Mac-1) [9-11], cellular protein kinases [12], reactive oxygen species (ROS) [13] and small guanosine triphosphatases (such as Rho, Rac) [9, 11]. Ligand binds with integrin Mac-1 to promote macrophages phagocytosis through Syk activation and NF- $\mathrm{KB}$ p65 translocation [14, 15]. Rho family GTPases regulated F-actin rearrangement, which promotes phagocytosis $[9,11]$. Mac-1 is a ligand of Intracellular adhesion molecule-1 (ICAM-1; CD54) [16], which plays an essential role in protein kinases $\mathrm{C}$ and Rho [17].

ICAM-1, a transmembrane glycoprotein, belongs to the immunoglobulin supergene family. It has been found expressed on various cells, such as endothelial and epithelial cells, smooth muscle cells, fibroblasts, and most leukocyte subsets including lymphocytes, neutrophils and monocytes/ macrophages [14, 18, 19]. Inflammatory cytokines, such as interleukin (IL)-1, tumor necrosis factor- $\alpha$ (TNF), interferon (IFN) $\gamma$ or with lipopolysaccharide (LPS) could increase ICAM-1 expression in multiple cell types $[14,17,20]$. Many studies have confirmed that the upregulated ICAM-1, especially on the resident cells (endothelial cells and epithelial cells), was involved in the process of inflammation, endotoxemia, and sepsis [21-25]. As an adhesion molecular, ICAM-1 plays a critical role in leukocyte trafficking and cell adhesion $[16,18]$. Interaction of ICAM-1 on endothelial cells and Mac-1/LFA-1 on leukocytes could trigger the localized endothelial cell cytoskeletal rearrangements and promote leukocyte-endothelial interactions [26]. Notably, with inflammatory stimuli, not only the ICAM-1 on the resident cell was upregulated, but also the ICAM-1 on macrophages. Under normal circumstances, ICAM-1 showed low expression on macrophages, while it was found upregulated under the inflammatory condition [27-30]. However, they mostly focus on the role of endothelial cells ICAM-1 or neutrophils ICAM-1, little is known about the function of macrophage-expressed ICAM-1. Previous studies found that ICAM-1 on endothelial cells mediated the cytoskeletal rearrangements of endothelia, which remind us to speculate that whether ICAM-1 on macrophages also participate in the macrophage phagocytosis.

In this study, we demonstrate that the increased ICAM-1 on macrophages enhances the phagocytosis under LPS stimulation. The mechanism of ICAM-1-mediated macrophage phagocytosis is depending on TLR4 signaling and cytoplasmic or mitochondrial reactive oxygen species (ROS) production. Therefore, our findings provided significant light on the regulation and phagocytosis of macrophage ICAM-1 and suggest that macrophage ICAM-1 contributes to against pathogens through phagocytosis.

\section{Materials and methods}

\section{Animals}

C57BL/6 male mice (6-8 weeks old) were obtained from Southern Medical University Animal Center (Guangzhou, China). TLR4 knockout mice (6-8 weeks old) were purchased from the Animal Core Facility of Nanjing Medical University (Nanjing, China). The experiments and animal care were performed in accordance with the guidelines of Southern Medical University.

\section{Reagents}

LPS (L3023), NAC (A7250) and apocynin (178385) were purchased from Sigma. MitoQ was from Focus Biomolecules (10-1363). Rabbit polyclonal antibody against GAPDH (5174) and Alexa Fluor ${ }^{\circledR} 555$ Phalloidin (8953) were from Cell Signaling Technology. ICAM-1 neutralizing antibody (YN1/1.7.4) (16-0541-85), Mouse IgG Isotype Control (02-6502), and pHrodo ${ }^{\circledR}$ Green dye conjugate (P35366) were obtained from Thermo Fisher Scientific. $\mathrm{H}_{2}$ DCFDA (ab113851) was obtained from Abcam. BUV395 Hamster Anti-Mouse ICAM-1 $(740,222)$ was obtained from BD Biosciences. Control siRNA (sc-37007), ICAM-1 siRNA (sc29355), and ICAM-1 antibody (sc-8439, sc-8439 PE) were purchased from Santa Cruz Biotechnology.

\section{Bone marrow-derived macrophages (BMDM) isolation and culture}

BMDM were isolated as described [31]. Briefly, bone marrow cells were flushed out from tibia and femur of mice and cultured in macrophage medium (DMEM containing 10\% FBS, complemented with $50 \mu \mathrm{g} / \mathrm{ml}$ penicillin/streptomycin and $10 \eta \mathrm{g} / \mathrm{ml} \mathrm{M-CSF}$ ) at $37{ }^{\circ} \mathrm{C}, 5 \% \mathrm{CO}_{2}$ for 7 days to differentiate into mature macrophages.

\section{Cell transfection}

For silence ICAM-1 expression, cells were transfected with si-NC and si-ICAM-1 by Lipofectamine ${ }^{\mathrm{TM}}$ LTX Reagent (Invitrogen) according to the manufacturer's instruction. After $48 \mathrm{~h}$, the silencing efficiency was measured by flow cytometry and immunoblotting.

\section{Quantification of phagocytosis}

BMDM cells were seeded in 6-well plates at a density of $2 * 10^{5}$ cells/well. After the various treatments, cells were incubated with pHrodo $^{\mathrm{TM}}$ Green E. coli BioParticles ${ }^{\mathrm{TM}}$ 
Conjugate $(0.5 \mu \mathrm{g} / \mathrm{ml})$ for $1 \mathrm{~h}$ at $37^{\circ} \mathrm{C}$ in the dark. The cells were collected and washed with PBS. The quantification of phagocytosis was analyzed by flow cytometry. Data were analyzed using FlowJo (TreeStar, USA).

\section{Flow cytometry assay}

To detect cell surface expression of ICAM-1, cells were incubated with BUV395 Hamster Anti-Mouse ICAM-1 (BD Biosciences) for $30 \mathrm{~min}$ on ice, washed three times with PBS, and analyzed by flow cytometry.

\section{Western blot}

The cells were lysed by RIPA buffer and the protein concentration of lysates was determined by BCA kit (Thermo Fisher Scientific). The proteins were separated by SDS-PAGE and transferred to PVDF membranes (Millipore). After blocking with $5 \%$ skimmed milk for $1 \mathrm{~h}$ at room temperature, the membrane was incubated with primary antibodies overnight at $4{ }^{\circ} \mathrm{C}$. After washing the membranes three times with TBST, the membranes were incubated with secondary antibody at room temperature for $1 \mathrm{~h}$, followed by visualization using ECL reagent (Millipore). The densitometric analysis of protein was performed by Image Pro Plus.

\section{Confocal microscopy}

Phagocytosis: BMDM cells were treated as required and incubated with $\mathrm{pHrodo}{ }^{\mathrm{TM}}$ Green E. coli BioParticles ${ }^{\mathrm{TM}}$ Conjugate $(0.5 \mu \mathrm{g} / \mathrm{ml})$ for $1 \mathrm{~h}$ at $37^{\circ} \mathrm{C}$ in the dark. After washed by PBS, cells were fixed with $4 \%$ paraformaldehyde (10 min at room temperature), permeabilized with $0.5 \%$ Triton X-100 in PBS (10 min) and block with $5 \%$ bovine serum albumin (BSA) for $1 \mathrm{~h}$. Then cells were incubated with Alexa Fluor 555 Phalloidin (Phalloidin can be used to describe the shape of the cell under confocal microscopy, 1:20) at $4{ }^{\circ} \mathrm{C}$ overnight. The nuclei were counterstained with DAPI (10 min) before images were taken on fluorescence microscope (Nikon Corporation).

ICAM-1 expression: Cells were seeded in glass slides. After necessary treatment, cells were fixed with $4 \%$ paraformaldehyde (10 min at room temperature) and block with $5 \%$ bovine serum albumin (BSA) for $1 \mathrm{~h}$. Then cells were incubated with ICAM-1 antibody PE $(1: 200)$ at $4{ }^{\circ} \mathrm{C}$ for $1 \mathrm{~h}$ at room temperature. Nuclei were counterstained with DAPI (10 min) before images were taken on fluorescence microscope (Nikon Corporation).

\section{Reactive oxygen species measurement}

BMDM cells at a density of $2 * 10^{5}$ cells/well in a 6 -well plate were cultured. After various treatments, cells were collected and stained cells in culture media with $20 \mu \mathrm{M}$ DCFDA (Abcam, ab113851) for $30 \mathrm{~min}$ at $37{ }^{\circ} \mathrm{C}$ in the dark. The cells were then washed twice with PBS after suspended in fresh DMEM, cells were analyzed by FACScan flow cytometry (BD Biosciences). Data were analyzed using FlowJo (TreeStar, USA).

\section{MnSOD and NADPH activity detection}

MnSOD activity was measured using the MnSOD Assay Kit with WST-8 (Beyotime Biotechnology, China) following the manufacturer's instructions. NADPH activity was measured by NADPH ELISA kit (Albion, China) following the manufacturer's instructions.

\section{Statistical analysis}

Results are given as mean $\pm \mathrm{SD}$. Comparisons between two groups was performed by the Student $t$-test, whereas multiple groups were assessed by the one-way ANOVA with SPSS20.0 statistic software. $P$ value $<0.05$ were considered statistically significant.

\section{Results}

\section{ICAM-1 is upregulated and correlated with enhanced phagocytosis on macrophage by LPS}

Although several studies have reported on the presence of ICAM-1 on macrophage[27, 29], little is known about the function of macrophage ICAM-1 under LPS stimulation. To investigate the role of LPS in ICAM-1 regulation, we compared the expression of ICAM-1 in BMDM after stimulating with or without LPS $(1 \mu \mathrm{g} / \mathrm{ml})$. We found that the percentage of ICAM-1-positive macrophages and the terms of mean fluorescence intensity (MFI) of ICAM-1 macrophages were significantly increased upon LPS stimulation in a timedependent manner (Fig. 1a-c). To confirm the flow analysis, ICAM cell surface expression was performed after exposure to LPS followed by confocal microscopy, which obtained the similar result (Fig. 1d). To further investigate the effect of LPS on ICAM-1, ICAM-1 expression was measured after LPS stimulation followed by western blot, which shows that LPS induced higher levels of ICAM-1 protein (Fig. 1e,f), suggesting that macrophages expression of ICAM-1 can be increased upon LPS stimulation at both the cell surface and protein levels.

Experimental study reported that ICAM-1 regulate cytoskeletal rearrangements of endothelia [17] and its ligand Mac-1 is a key regulator of phagocytosis [15], we hypothesized that macrophage ICAM-1 might be required for LPSinduced macrophage phagocytosis. To test this hypothesis, 
a

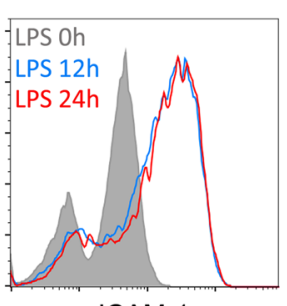

ICAM-1
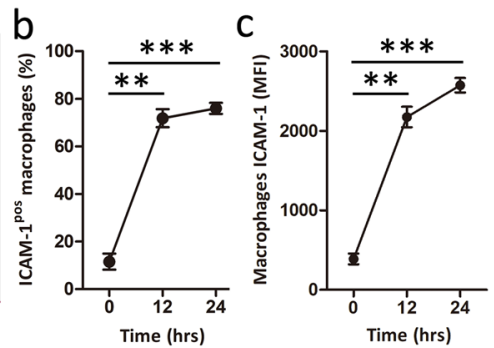

d
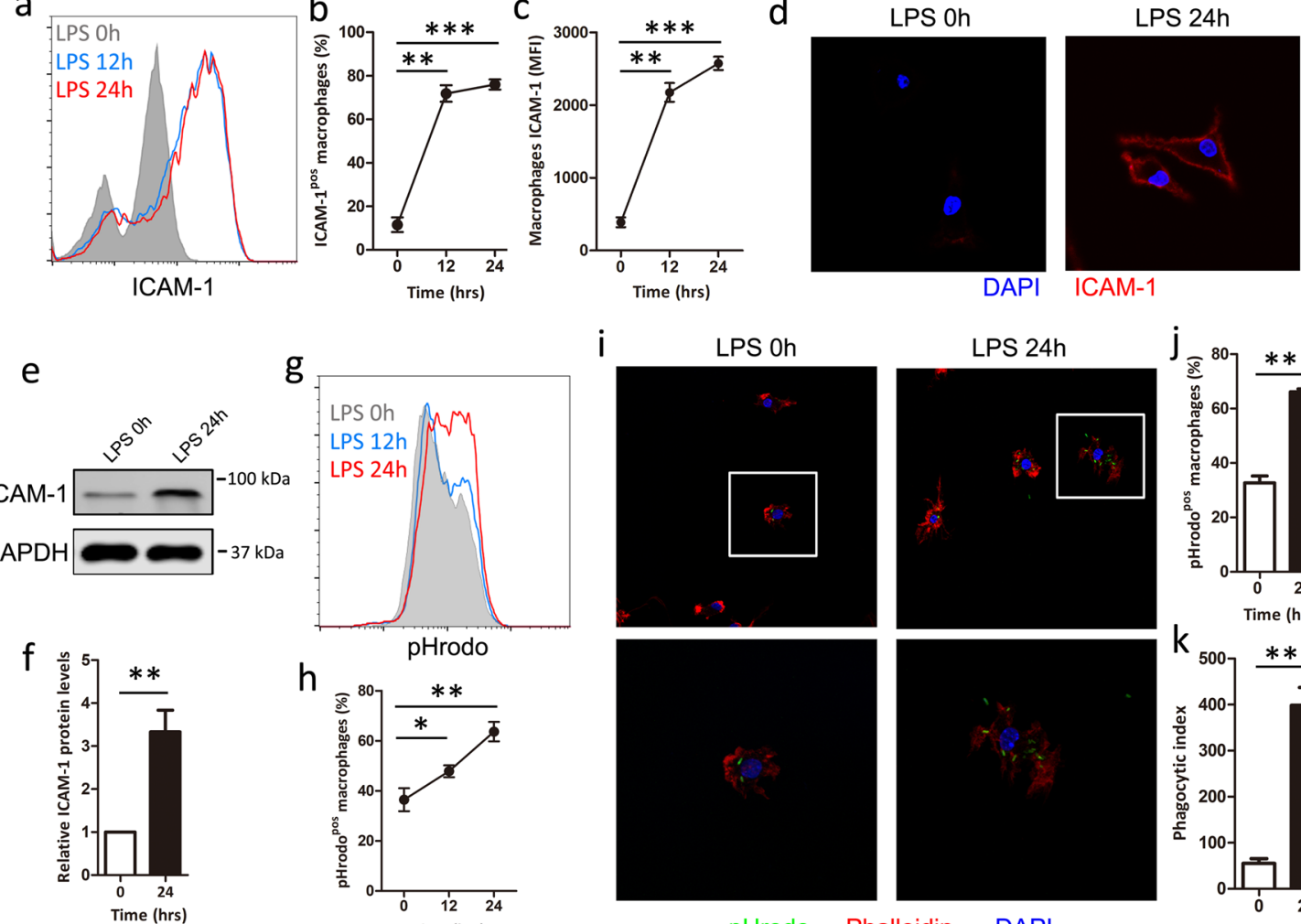

i

g
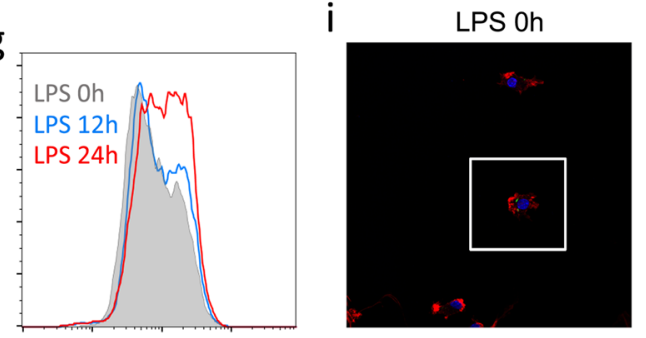

h

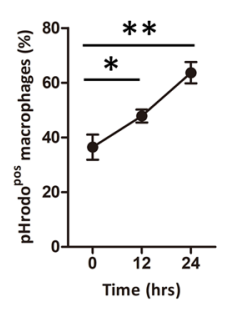

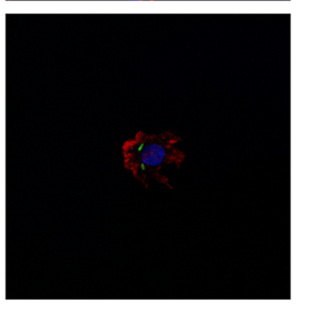

pHrodo Phalloidin
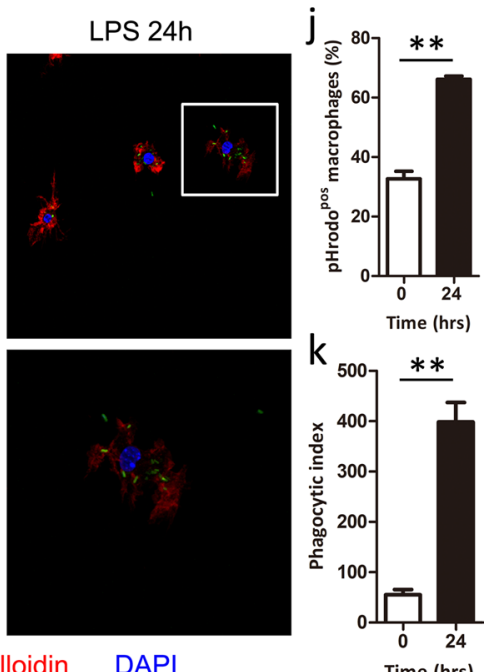

।

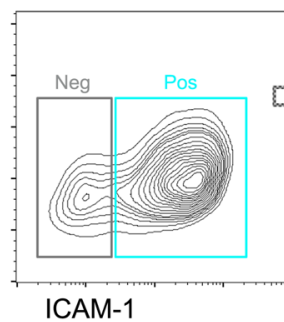

ICAM-1
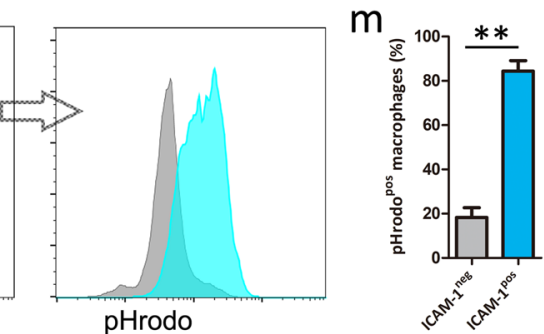

Fig. 1 LPS increases ICAM-1 expression and related to phagocytosis in macrophage. BMDM cells were treated with LPS $(1 \mu \mathrm{g} / \mathrm{ml})$ at the indicated time. (a-c) ICAM-1 cell surface expression was analyzed by flow cytometry. a Representative histogram of ICAM-1 cell surface expression. b Percentage of ICAM-1-positive (ICAM$1^{\text {pos }}$ ) macrophage is shown. c Mean fluorescence intensity (MFI) is shown. (d) ICAM-1 cell surface expression was measured by confocal microscopy. e, f BMDM cells were treated with or without LPS for $24 \mathrm{~h}$. e ICAM-1 protein expression was analyzed by western blot. GAPDH was used as a loading control. $\mathbf{f}$ Quantification of ICAM-1 expression. g, h BMDM cells were treated with LPS at the indicated time. Macrophage phagocytosis was analyzed using pHrodo by flow cytometry. g Representative histogram illustrating the detection of

we measured the macrophage phagocytosis using pHrodo by flow cytometry and confocal microscopy. Flow analysis showed that LPS significantly increased the percentage of pHrodo-positive macrophages ( $\mathrm{pHrodo}^{\mathrm{pos}}$ ) in a time-dependent manner (Fig. 1g, h). Confocal microscopy also showed similar results, which displays LPS significantly increased
pHrodo associated macrophages. h Percentage of pHrodo-positive (pHrodo $^{\text {pos }}$ ) macrophages is shown. $\mathbf{i}-\mathbf{k}$ BMDM cells were treated with or without LPS for $24 \mathrm{~h}$. Macrophage phagocytosis was analyzed using pHrodo by confocal microscopy. i Representative image of macrophage phagocytosis. $\mathbf{j}$ Percent of cells that displayed active phagocytosis was calculated. k Phagocytic index levels are shown. The phagocytic index levels indicate the average number of targets per 200 cells. $\mathbf{l}-\mathbf{m}$ Frequency of pHrodo positive macrophages within the ICAM-1 positive (ICAM- ${ }^{\text {pos}}$ ) or negative (ICAM- $1^{\text {neg }}$ ) population in LPS stimulated BMDM cells. Each bar represents the mean \pm SD based on three independent experiments. $* P<0.05$, $* * P<0.01, * * * P<0.001$

macrophage phagocytosis as measured by both the percentage of macrophages ingesting targets (\% phagocytosis) and the number of ingested opsonized targets per phagocytic cell (phagocytic index) (Fig. 1i-k). Interestingly, ICAM1-positive macrophages exhibited a greater phagocytosis (pHrodo $^{\text {pos }}$ ) than ICAM-1 negative cells, suggesting that 
the relation between ICAM-1 and macrophage phagocytosis (Fig. 11, m). Collectively, these results suggest the potential involvement of ICAM-1 in the regulation of LPS-induced macrophage phagocytosis.

\section{LPS-induced macrophage phagocytosis is dependent on ICAM-1}

To confirm whether ICAM-1 plays a role in the regulation of LPS-induced macrophage phagocytosis, we used ICAM-1 neutralizing antibody (anti-ICAM-1 mAb) to block the function of macrophage cell surface ICAM-1. We found that surface blockade of ICAM-1 decreased the percentage of pHrodo-positive macrophages (Fig. 2a, b) by flow cytometry. To confirm the flow analysis, the effect of ICAM-1 on macrophage phagocytosis was detected by confocal microscopy. We found that anti-ICAM-1 mAb inhibited the LPSinduced macrophage phagocytosis (Fig. 2c-e). These results suggest that cell surface blockade of ICAM-1 suppresses LPS-induced macrophage phagocytosis.

To further investigate the role of ICAM-1 in phagocytosis, we compared the efficient macrophage phagocytosis in si-NC and si-ICAM-1 cells after LPS stimulated. As shown in Fig. 3a and b, si-ICAM-1 showed significantly down-regulated the expression of ICAM-1 protein, compared with si-NC cells. Flow analysis showed that LPSinduced phagocytosis was significantly decreased in ICAM-1 silenced cells, compared with si-NC cells (Fig. 3c, d). In parallel, confocal microscopy analysis also showed similar results, knockdown of ICAM-1 significantly suppressed the efficient macrophage phagocytosis (Fig. 3e-g). Taken together, these results suggest that ICAM- 1 is required for LPS-induced macrophage phagocytosis.

\section{ICAM-1 regulates LPS-induced macrophage phagocytosis, is TLR4-dependent}

Having identified a role for ICAM-1 in macrophage phagocytosis, the potential associated mechanisms were next investigated. Since the corresponding receptor of LPS is TLR4, which has been reported as a regulator of phagocytosis [32] we, therefore, investigated whether TLR4 could regulate ICAM-1 expression at both cell surface and protein in LPS-induced macrophage phagocytosis. Our results also indicated that TLR4 was essential for LPS-induced macrophage phagocytosis (Fig. 4a, b). Next, we examined ICAM-1 cell surface expression and total protein in macrophage from WT or TLR4 knockout macrophage with or a

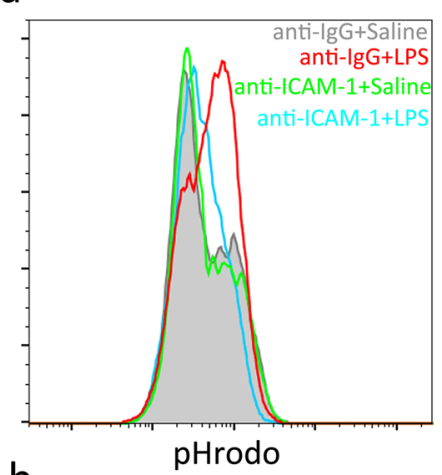

b

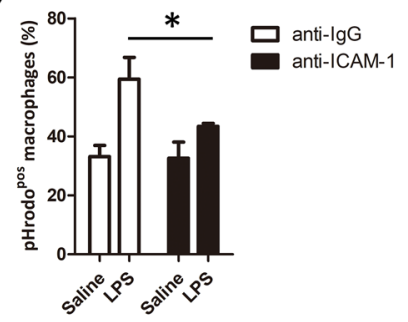

c
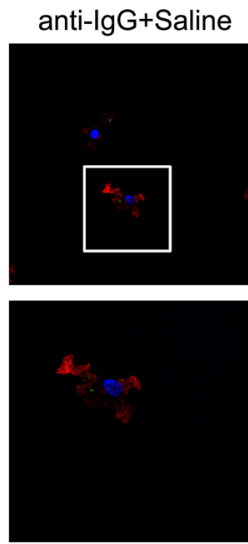

d

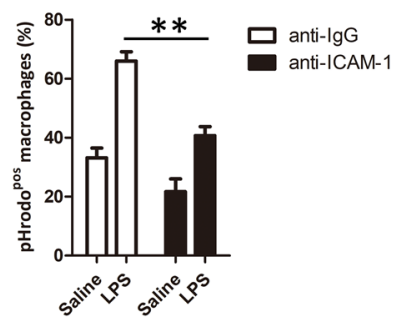

anti-lgG+LPS
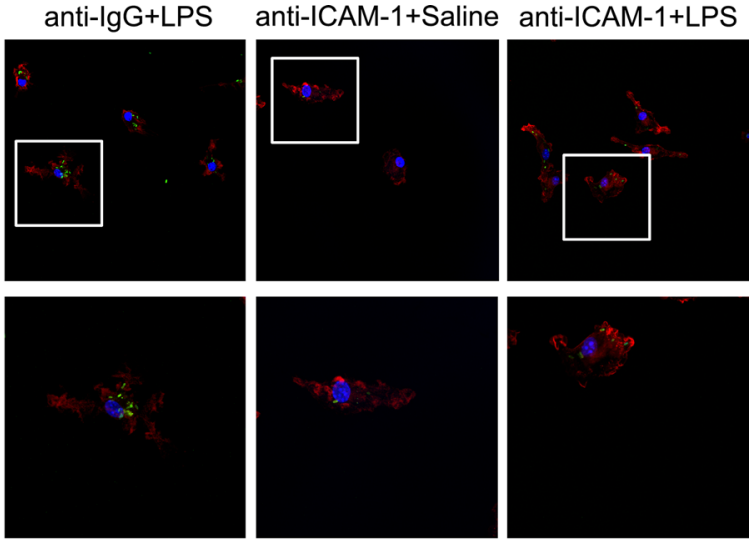

Phalloidin

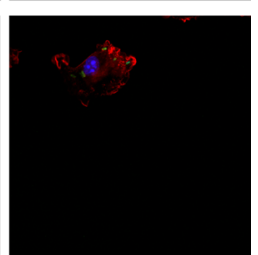

DAPI

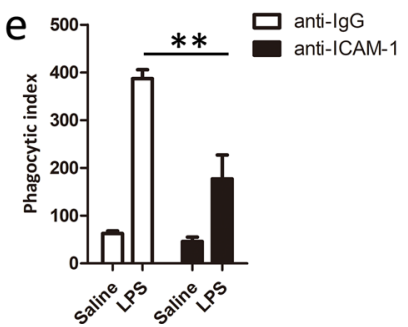

Fig. 2 ICAM-1 neutralizing antibody attenuates LPS-induced phagocytosis in macrophage. BMDM cells were pretreated with or without neutralizing antibodies against ICAM-1 for $15 \mathrm{~min}$ and then exposed to LPS $(1 \mu \mathrm{g} / \mathrm{ml})$ for $24 \mathrm{~h}$. a, b Macrophage phagocytosis was analyzed using pHrodo by flow cytometry. a Representative histogram illustrating the detection of pHrodo associated macrophages. b Per- centage of pHrodo-positive ( $\mathrm{pHrodo}^{\mathrm{pos}}$ ) macrophages is shown. c-e Macrophage phagocytosis was analyzed using pHrodo by confocal microscopy. c Representative image of macrophage phagocytosis. Percentage of phagocytosis (d) and Phagocytic index levels (e) are shown. Each bar represents the mean \pm SD based on three independent experiments. $* P<0.05$, $* * P<0.01$ 

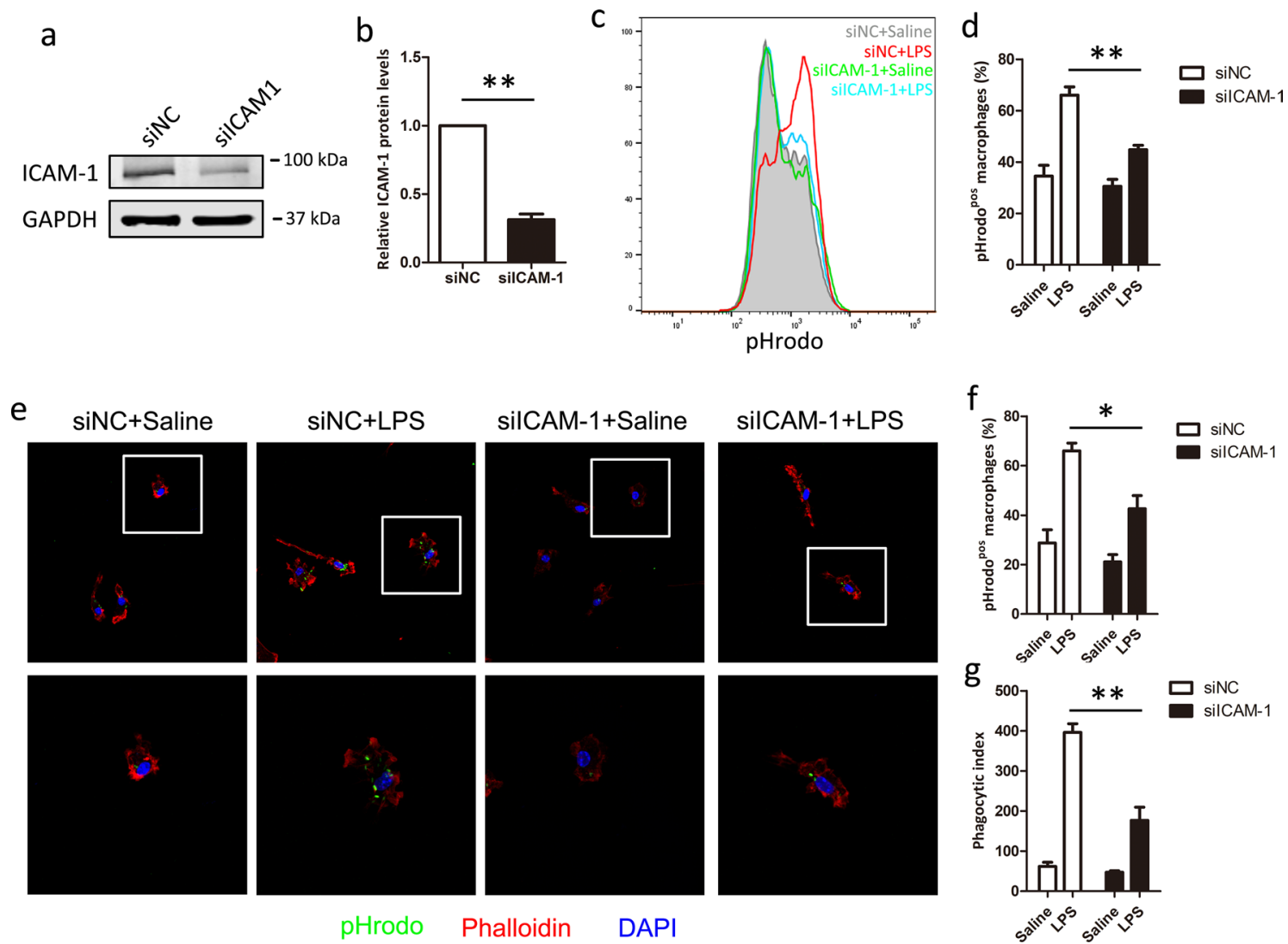

Fig. 3 Silencing of ICAM-1 expression reduces LPS-induced phagocytosis in macrophages. BMDM cells were transfected with si-NC or si-ICAM-1 for $48 \mathrm{~h}$. a, b The efficiency of silencing was evaluated by western blot after $48 \mathrm{~h}$. GAPDH was used as quantitative controls. BMDM cells transfected with si-NC or si-ICAM-1 were treated with LPS for $24 \mathrm{~h}$ to assay the macrophage phagocytosis. c, $\mathbf{d}$ Macrophage phagocytosis was analyzed using pHrodo by flow cytometry. c Rep-

without LPS stimulation. We found that TLR4 knockout macrophage had no impact on ICAM-1 expression at both cell surface and total protein (Fig. 4c-f) on normal condition. However, the cell surface expression of ICAM-1 upon LPS stimulation was significantly inhibited in TLR4 knockout macrophage, compared with WT macrophage (Fig. 4c-e). Furthermore, as shown in Fig. 4f and g, TLR4 knockout macrophage also showed decreased ICAM-1 protein expression after LPS stimulation by western blot analysis. These data suggest that LPS-induced macrophage phagocytosis is dependent on TLR4-mediated ICAM-1 expression.

\section{ROS production is required for TLR4-mediated ICAM-1 expression}

Because ROS is involved in phagocytic process and TLR4 signaling [13], we hypothesized that ROS might contribute to TLR4-mediated ICAM-1 expression in macrophage. We measured the change of ROS in BMDM cells after resentative histogram illustrating the detection of pHrodo associated macrophages. d Percentage of pHrodo-positive (pHrodo ${ }^{\mathrm{pos}}$ ) macrophages is shown. $\mathbf{e}-\mathbf{g}$ Macrophage phagocytosis was analyzed using pHrodo by confocal macroscopy. e Representative image of macrophages phagocytosis. Percentage of phagocytosis (f) and Phagocytic index levels $(\mathbf{g})$ are shown. Each bar represents the mean \pm SD based on three independent experiments. $* P<0.05, * * P<0.01$

LPS treatment using redox-sensitive fluorescent probe 2',7'-Dichlorofluorescein diacetate (DCFH2-DA). As shown in Fig. 5a and b, ROS was dramatically increased in response to LPS treatment, indicating that oxidative stress occurred in LPS treated BMDM cells. Then, cells were pretreated with antioxidant, NAC, which degrades intracellular ROS, before LPS treatment. We found NAC pretreatment almost fully reversed the LPS-induced phagocytosis and ROS outburst (Fig. 5a-d). Moreover, we found that the production of ROS upon LPS stimulation was significantly decreased in TLR4 knockout macrophage, compared with WT macrophage (Fig. 5e, f). To establish the role for ROS in macrophage ICAM-1 expression by LPS, we measured ICAM-1 protein levels and cell surface expression, pretreatment with NAC, follow by LPS stimulation. We found that NAC effectively decreased the percentage of ICAM-1-positive macrophage and terms of mean fluorescence intensity (MFI) of ICAM-1 on macrophage, indicating that ROS regulated ICAM-1 cell surface expression (Fig. 5g-i). Furthermore, as shown in 
a

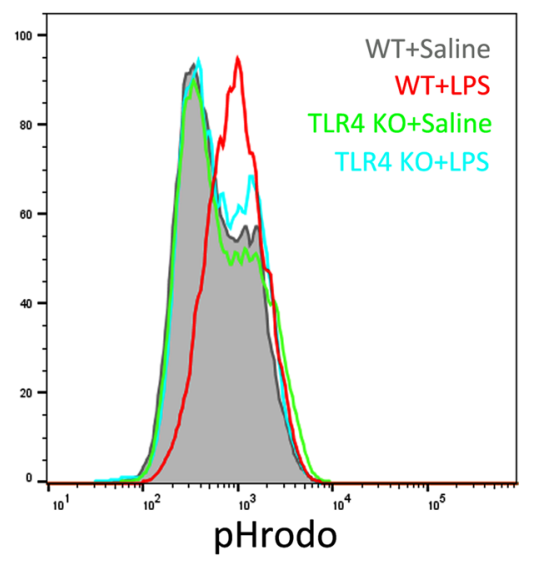

b

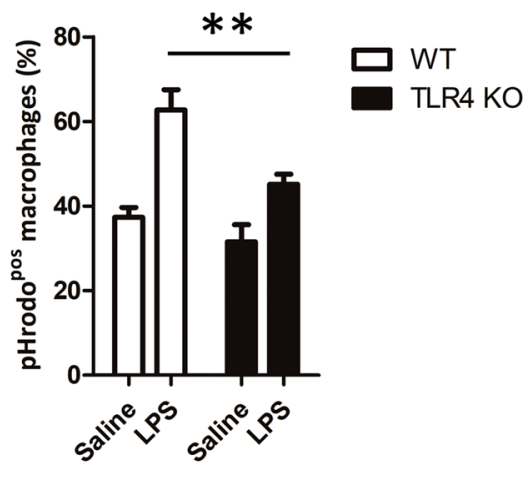

C

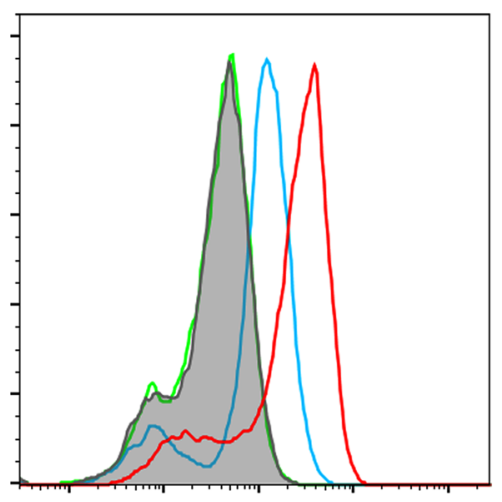

ICAM-1

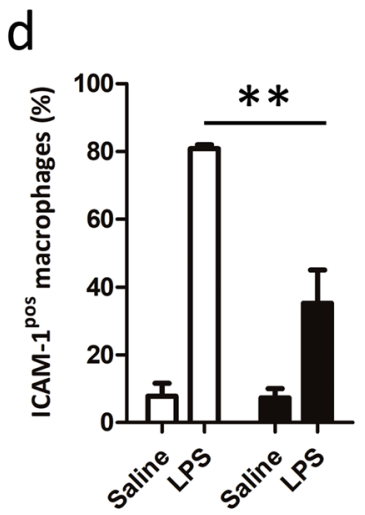

e

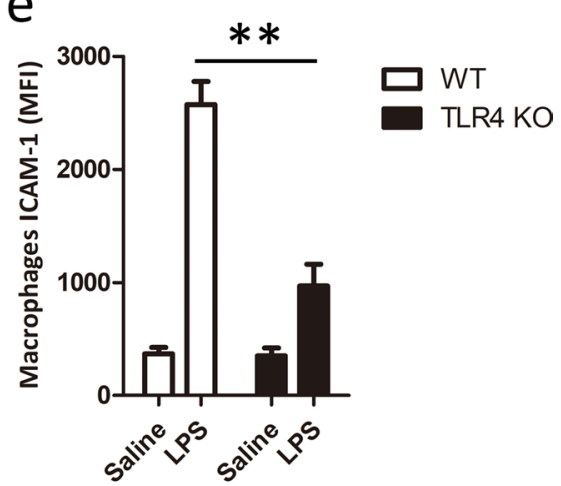

WT+Saline WT+LPS TLR4 KO+Saline TLR4 KO+LPS

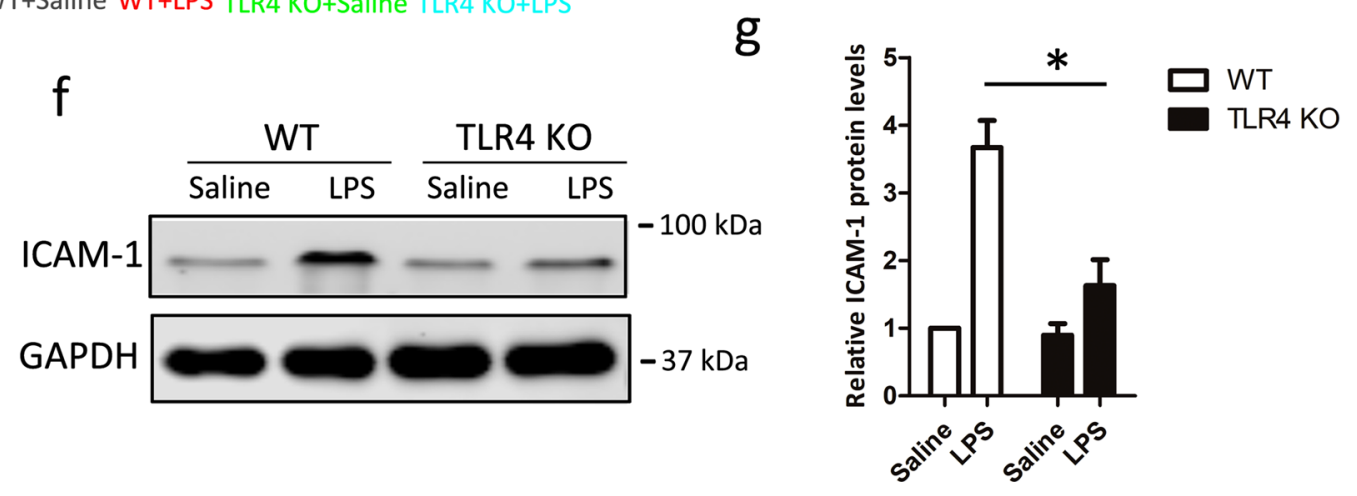

Fig. 4 TLR4 regulates ICAM-1 expression upon LPS stimulation. LPS stimulated BMDM cells from WT and TLR4 knockout mice for $24 \mathrm{~h}$. Macrophage phagocytosis and ICAM-1 cell surface expression were analyzed by flow cytometry. a Representative histogram illustrating the detection of pHrodo associated macrophages. b Percentage of pHrodo-positive (pHrodo ${ }^{\mathrm{pos}}$ ) macrophages is shown. $\mathbf{c}$ Representa-

Fig. 5j and k, NAC also showed decreased ICAM-1 protein levels after LPS stimulation by western blot analysis, indicating that ROS also regulated ICAM-1 protein levels. To confirm the origin of ROS, we measure the activity of NADPH or Mn-SOD, which indicates intracellular ROS or mitochondria ROS, respectively. We found that LPS could increase NADPH or Mn-SOD activity and TLR4 knockout tive histogram of ICAM-1 cell surface expression. d Percentage of ICAM-1-positive (ICAM-1 ${ }^{\text {pos}}$ ) macrophages is shown. e Mean fluorescence intensity (MFI) is shown. f ICAM-1 total protein expression was measure by western blot. GAPDH was used as a loading control. g Quantification of ICAM-1 expression. Data shown represent three independent experiments, ${ }^{*} P<0.05$, $* * P<0.01$

can inhibit NADPH and Mn SOD activity after LPS stimulation (Fig. 51 and m). Besides, apocynin (inhibit NADPH oxidases) or MitoQ (degrade mitochondria ROS) pretreatment could suppress the overproduction of ROS after LPS stimulation (Fig. 5n and o). Furthermore, Apocynin and MitoQ pretreatment could inhibit ICAM-1 cell surface expression (Fig. 5p and q) and the protein levels (Fig. 5r and 


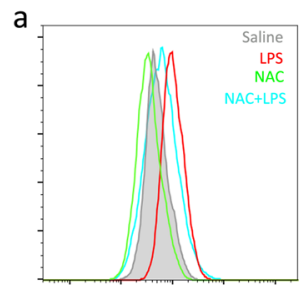

b

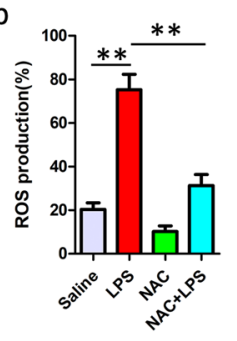

e

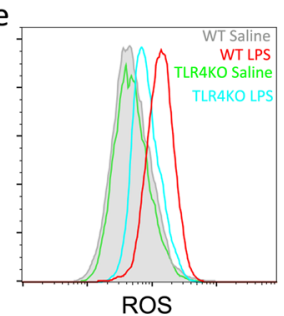

$f$

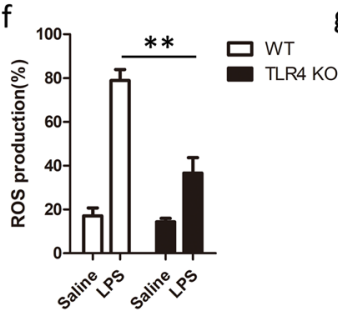

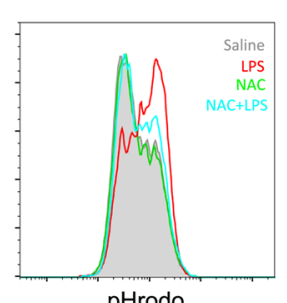

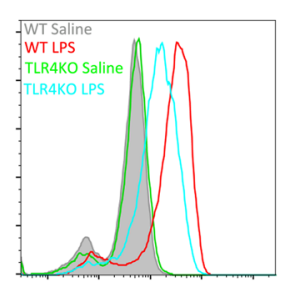

d

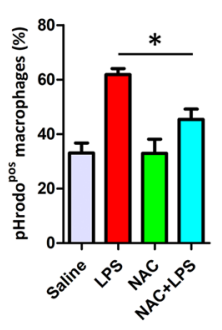

$\mathrm{h}$

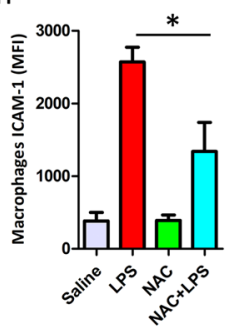

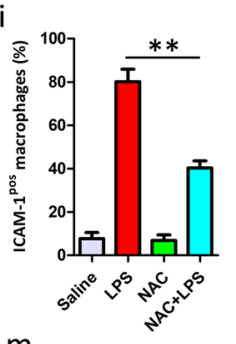

$\mathrm{m}$

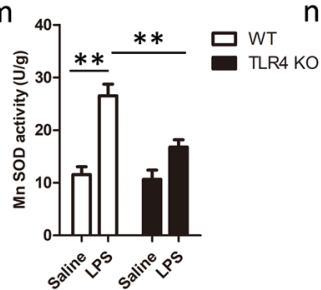

q

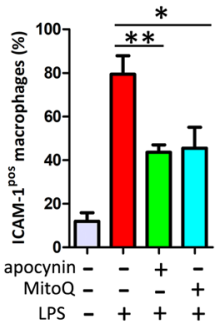

j

ICAM-1
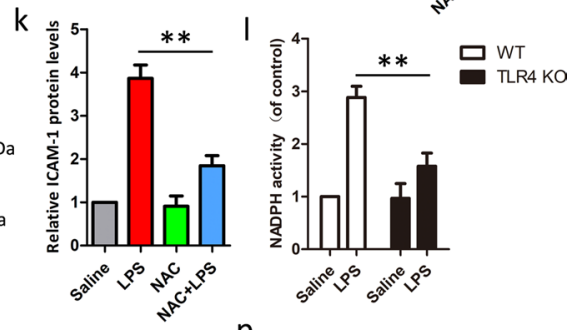

$p$

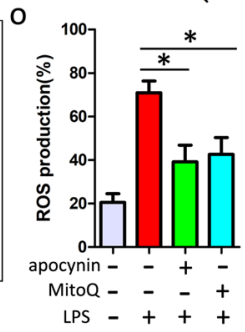

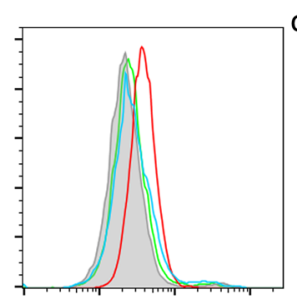

ROS

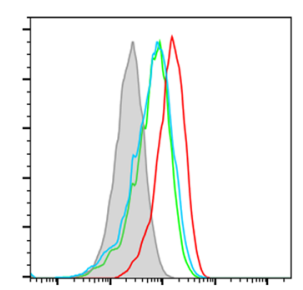

ICAM-1

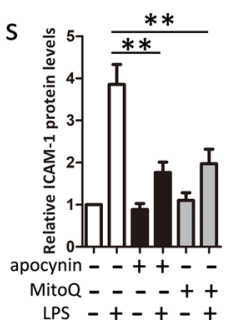

Fig. 5 Inhibition of ROS reduces LPS-induced macrophage ICAM-1 expression and phagocytosis. BMDM cells were pretreated with or without $5 \mathrm{mM}$ NAC, $5 \mu \mathrm{M}$ apocynin, or $10 \mathrm{nM}$ MitoQ for $1 \mathrm{~h}$ before LPS $(1 \mu \mathrm{g} / \mathrm{ml}, 24 \mathrm{~h})$ treatment. Macrophage phagocytosis and ROS production were measured by flow cytometry. a Representative histogram of ROS production. b Percentage of ROS-positive (ROS ${ }^{\text {pos}}$ ) macrophages is shown. c Representative histogram illustrating the detection of pHrodo associated macrophages. d Percentage of pHrodo-positive ( pHrodo $^{\text {pos }}$ ) macrophages is shown. e, f The production of ROS was measured in LPS-stimulated BMDM from WT and TLR4 knockout mice. e Representative histogram of ROS production is shown. $\mathbf{f}$ Percentage of ROS-positive $\left(\mathrm{ROS}^{\mathrm{pos}}\right)$ macrophages is shown. g-i BMDM cells were pretreated with NAC $1 \mathrm{~h}$ before LPS $(1 \mu \mathrm{g} / \mathrm{ml})$ treatment. ICAM-1 cell surface expression was analyzed by flow cytometry. $\mathbf{g}$ Representative histogram of ICAM-1 cell surface expression. h Percentage of ICAM-1-positive (ICAM- ${ }^{\text {pos }}$ ) macrophages is shown. i Mean fluorescence intensity (MFI) is shown. $\mathbf{j}$ ICAM-1 total protein level is measure by western blot. GAPDH was used as a loading control. k Quantification of ICAM-1 expression. I ELISA measured NADPH activity. $\mathbf{m}$ WST-8 assessed MnSOD activity. n ROS production was measured by flow cytometry. o Percentage of ROS-positive (ROS ${ }^{\text {pos }}$ ) macrophages is shown. p ICAM-1 cell surface expression was measured by flow cytometry. q Percentage of ICAM-1-positive (ICAM-1 ${ }^{\text {pos }}$ ) macrophages is shown. $\mathbf{r}$ ICAM-1 total protein level is measure by western blot. GAPDH was used as a loading control. s Quantification of ICAM-1 expression. Data shown represent three independent experiments, ${ }^{*} P<0.05$, ${ }^{*} P<0.01$ 
s), indicating ROS (both NADPH-derived and mitochondria-derived ROS) contributes to ICAM-1 expression. Taken together, these results suggest that TLR4-mediated ROS production is required for ICAM-1 expression in macrophage.

\section{Discussion}

Endotoxemia, a systemic inflammatory response induced by infection, can result in multiple organ dysfunction and death [1-3]. Despite many advances in treatment, such as antibiotic therapy, ventilator management, resuscitative strategies, and blood glucose maintenance, sepsis or medotoxemia is still one of the main causes of death in critical patients [31, 33, 34]. The innate immune system, the first response to infection, plays a critical role in the initiation and development of sepsis $[4,5]$. Phagocytosis plays a critical role in that process. Hence, it is essential for us to understand the underlying pathogenetic mechanisms of macrophage phagocytosis in endotoxemia or sepsis. Therefore, in this study, we identified the function and mechanism of ICAM-1 in LPS-induced macrophage phagocytosis.

ICAM-1 plays an important role in leukocyte trafficking, immunological synapse formation and numerous cellular immune responses [16, 17, 35]. ICAM-1 is now known expressed on essentially most leukocyte subsets (such as neutrophils and macrophages/monocyte), endothelial cells, epithelial cells, fibroblasts and others. Under non-inflammatory conditions, ICAM-1 expression is constitutively low on most cell types, including macrophage [14, 29]. However, under inflammatory conditions (such as TNF- $\alpha$, IL-1 $\beta$, IFN- $\gamma$ stimulation), ICAM-1 is upregulated, particularly on endothelium, which regards as a marker of pro-adhesive state in endothelial cells $[17,30]$. Besides, in sepsis, upregulation of ICAM-1 in organs had been reported in many studies [21, 24]. Several studies used anti-ICAM-1 antibodies or gene deficiency animals to investigate the direct role of ICAM-1 in sepsis, but inconsistent results were found among them $[23,25]$. Some studies reported that blockade of ICAM-1 is beneficial for the septic animals, while others revealed that blockade of ICAM-1 decreased the survival rate in the sepsis model $[22,24,36]$. The direct role of ICAM-1 in sepsis still remained controversial, but these studies revealed that ICAM-1 play a critical role in sepsis. However, the mechanism they focus on are soluble ICAM-1, endothelial ICAM-1 or neutrophil ICAM-1, the knowledge about the function of macrophage-expressed ICAM-1 is limited.

At present, ICAM-1 is not commonly thought as a macrophage cell surface antigen, but it can be upregulated in macrophage under numerous infectious and inflammatory settings. Early studies have reported that ICAM-1 expression was significantly increased in bone marrow-derived macrophage, human macrophage cell line THP1, or peritoneal macrophage after M. tuberculosis, mycobacterial or BCG infection [37-40]. The surface expression and mRNA levels of ICAM1 in macrophage can be detected in microgravity experiments [41]. Furthermore, inflammatory factors, such as TNF-a, LPS, stimulate ICAM-1 expression in mouse macrophages [29]. Although several studies have reported that the relationship between ICAM-1 and macrophage polarization [27, 28], little know about others function of ICAM-1 on macrophage under LPS stimulation. In our results, the cell surface expression of ICAM-1 was increased in LPS-stimulated macrophage. Besides, we found that ICAM-1 protein levels also significantly increased after LPS stimulation. Since LPS is the major component of Gram-negative bacteria, and Mac-1, a ligand of ICAM-1 [16], involve in phagocytosis, we hypothesized that elevated macrophage ICAM-1 may associated with phagocytosis. Indeed, we found that ICAM-1-positive macrophages exhibited a greater phagocytosis than ICAM-1 negative cells, which indicates that ICAM-1 could enhance the macrophage phagocytosis.

Phagocytosis is a complex cellular process that is a key role in innate immunity. To investigate the relationship between ICAM-1 and macrophage phagocytosis, we inhibited ICAM-1 by neutralizing antibody or silenced its expression by siICAM-1. We found that macrophage phagocytosis was significantly decreased by inhibited ICAM-1, suggesting ICAM-1 is required for macrophage phagocytosis. Study has shown that the C-terminal domain of ICAM-1 could interact with cellular a-actinins or filamin B [42], which promotes cytoskeletal rearrangements, which is necessary for phagocytosis. Besides, the cytoplasmic tyrosine kinase Syk [12] and small guanosine triphosphatases Rho $[9,11]$ have been link to macrophage phagocytosis and could be activated by ICAM-1, which provides the downstream mechanism of ICAM-1-mediate macrophage phagocytosis. Of note, inhibiting of macrophage ICAM-1 did not affect the phagocytosis without LPS stimulation, indicating that ICAM-1-dependent macrophage phagocytosis requires activated macrophage by LPS.

As we all know, LPS is a ligand of TLR4 and TLR4 signaling activates macrophage phagocytosis, so we explored the relationship between TLR4 and ICAM-1 in macrophage. Although several studies have reported that TLR4 regulated ICAM-1 expression in many cell types, such as aortic valve interstitial cells [43, 44], coronary artery endothelial cells [45], pulmonary vascular endothelial cells [46] and neutrophils [47], little is known about the relationship between ICAM- 1 and macrophage. Therefore, in this study, we found that ICAM-1 cell surface expression was decreased in TLR4 knockout macrophage after LPS stimulation at both cell surface expression and total protein levels, suggesting that TLR4 regulates ICAM-1 expression in macrophage. In addition, 
the TLR4-dependent activation of phagocytosis was inhibited by neutralizing or silencing of ICAM-1, providing that TLR4-dependent activation of phagocytosis requires signaling through ICAM-1. Because ROS contributes to macrophage phagocytosis and TLR4 signaling [32], we hypothesized that ROS production by LPS/TLR4 signaling associated with ICAM-1 expression in macrophage. Indeed, we observed high levels of ROS in LPS-stimulated WT cells, which could be decreased in TLR4 knockout macrophage. Besides, decreasing the production of ROS by NAC can inhibit the cell surface expression of ICAM-1, indicating ROS production triggered by LPS/TLR4 signaling associated with ICAM-1 expression in macrophage. The production of ROS by macrophage majorly relies on Nox 2 gene, which could be regulated by TLR4-dependent PI3K signaling [48]. In this study, we found that not only cytoplasmic ROS but also mitochondrial ROS induced increasing ICAM expression in macrophage. Other studies have shown that ICAM-1 expression is mediated by PI3K $[49,50]$. We suppose that the detailed mechanism may be due to PI3K signaling activated triggered by LPS/TLR4 signaling causes Nox 2 gene or mitochondrial induces ROS production, which leads to the increase of ICAM-1 expression resulting in Syk or Rho activated, which in turn regulates macrophage phagocytosis. It has been shown that ICAM-1-mediated endocytosis is clathrin- and caveolarindependent in several cell types, yet it involves cytoskeletal reorganizations similar to macropinocytosis [51], can support uptake of large micrometer sized particles [52], depend on acid sphingomyelinase production of ceramide at the cell surface in areas of endocytosis [53], but the detail mechanism is unclear, therefore, further studies are required to further illuminate the mechanistic details of ICAM-1-dependent macrophage phagocytosis.

In conclusion, we have identified the effect of ICAM-1 in mediating LPS-induced macrophage phagocytosis and the mechanism of ICAM-1-mediated macrophage phagocytosis is depending on TLR4-mediated cytoplasmic and mitochondrial ROS production. Therefore, our findings provided significant light on the expression of macrophage ICAM-1, maybe enhanced in endotoxemia or sepsis through phagocytosis.

Acknowledgements This work was supported by the National Natural Science Foundation of China (No. 81671957 and 81873951), Key projects of Guangdong Natural Science Foundation (No. 2018B030311038), Science and Technology Planning Project of Guangdong Province (No. 2016A020215212), Science and Technology Program of Guangzhou (No.201904010382). We thank Xuegang Sun, Zaisheng Qin for their discussion and Yuanliang Liu and Zhiyun Zeng for their technical assistance.

Author contributions J. T. and J. L. devised the concept, designed the research, supervised the study, and wrote the paper. H. Z. performed experiments and analyzed data with J. T., who also prepared the figures.
H. L., Q. P., J. Z., X. Liu, and X. Li performed the experiments. L. Z. revised the manuscript. All authors read and approved the final manuscript.

\section{Compliance with ethical standards}

Conflict of interest The authors declare that they have no known competing financial interests or personal relationships that could have appeared to influence the work reported in this paper.

\section{References}

1. Shankar-Hari M, Phillips GS, Levy ML, Seymour CW, Liu VX, Deutschman CS, et al. Developing a new definition and assessing new clinical criteria for septic shock: for the third international consensus definitions for sepsis and septic shock (Sepsis-3). JAMA. 2016;315:775-87.

2. Kaukonen KM, Bailey M, Pilcher D, Cooper DJ, Bellomo R. Systemic inflammatory response syndrome criteria in defining severe sepsis. N Eng J Med. 2015;372:1629-38.

3. Seymour CW, Liu VX, Iwashyna TJ, Brunkhorst FM, Rea TD, Scherag A, et al. Assessment of clinical criteria for sepsis: for the third international consensus definitions for sepsis and septic shock (Sepsis-3). JAMA. 2016;315:762-74.

4. Hirayama D, Iida T, Nakase H. The phagocytic function of macrophage-enforcing innate immunity and tissue homeostasis. Int J Mol Sci 2017; 19.

5. Murray PJ, Wynn TA. Protective and pathogenic functions of macrophage subsets. Nat Rev Immunol. 2011;11:723-37.

6. Aderem A. Phagocytosis and the inflammatory response. J Infect Dis. 2003;187(Suppl 2):S340-5.

7. Rougerie P, Miskolci V, Cox D. Generation of membrane structures during phagocytosis and chemotaxis of macrophages: role and regulation of the actin cytoskeleton. Immunol Rev. 2013;256:222-39.

8. Aderem A, Underhill DM. Mechanisms of phagocytosis in macrophages. Annu Rev Immunol. 1999;17:593-623.

9. Hall AB, Gakidis MA, Glogauer M, Wilsbacher JL, Gao S, Swat $\mathrm{W}$, et al. Requirements for Vav guanine nucleotide exchange factors and Rho GTPases in FcgammaR- and complement-mediated phagocytosis. Immunity. 2006;24:305-16.

10. Kaplan G. Differences in the mode of phagocytosis with Fc and C3 receptors in macrophages. Scand J Immunol. 1977;6:797-807.

11. Tzircotis G, Braga VM, Caron E. RhoG is required for both FcgammaR- and CR3-mediated phagocytosis. J Cell Sci. 2011;124:2897-902.

12. Cheeseman KL, Ueyama T, Michaud TM, Kashiwagi K, Wang D, Flax LA, et al. Targeting of protein kinase C-epsilon during Fcgamma receptor-dependent phagocytosis requires the epsilonC1B domain and phospholipase C-gamma1. Mol Biol Cell. 2006;17:799-813.

13. Brown KL, Christenson K, Karlsson A, Dahlgren C, Bylund J. Divergent effects on phagocytosis by macrophage-derived oxygen radicals. J Innate Immunity. 2009;1:592-8.

14. Woodfin A, Beyrau M, Voisin MB, Ma B, Whiteford JR, Hordijk PL, et al. ICAM-1-expressing neutrophils exhibit enhanced effector functions in murine models of endotoxemia. Blood. 2016;127:898-907.

15. Liu YS, Wang LF, Cheng XS, Huo YN, Ouyang XM, Liang LY, et al. The pattern-recognition molecule mindin binds integrin Mac-1 to promote macrophage phagocytosis via Syk 
activation and NF-kappaB p65 translocation. J Cell Mol Med. 2019;23:3402-16.

16. Diamond MS, Staunton DE, de Fougerolles AR, Stacker SA, GarciaAguilar J, Hibbs ML, et al. ICAM-1 (CD54): a counter-receptor for Mac-1 (CD11b/CD18). J Cell Biol. 1990;111:3129-39.

17. Hubbard AK, Rothlein R. Intercellular adhesion molecule-1 (ICAM1) expression and cell signaling cascades. Free Radical Biol Med. 2000;28:1379-86.

18. Proebstl D, Voisin MB, Woodfin A, Whiteford J, D'Acquisto F, Jones GE, et al. Pericytes support neutrophil subendothelial cell crawling and breaching of venular walls in vivo. J Exp Med. 2012;209:1219-34.

19. Springer TA. Adhesion receptors of the immune system. Nature. 1990;346:425-34.

20. Roebuck KA, Finnegan A. Regulation of intercellular adhesion molecule-1 (CD54) gene expression. J Leukoc Biol. 1999;66:876-88.

21. Glushakova O, Kosugi T, Roncal C, Mu W, Heinig M, Cirillo P, et al. Fructose induces the inflammatory molecule ICAM-1 in endothelial cells. J Am Soc Nephrol. 2008;19:1712-20.

22. Zhao YJ, Yi WJ, Wan XJ, Wang J, Tao TZ, Li JB, et al. Blockade of ICAM-1 improves the outcome of polymicrobial sepsis via modulating neutrophil migration and reversing immunosuppression. Mediators Inflamm. 2014;2014:195290.

23. van Griensven M, Probst C, Muller K, Hoevel P, Pape HC. Leukocyte-endothelial interactions via ICAM-1 are detrimental in polymicrobial sepsis. Shock (Augusta, Ga). 2006;25:254-9.

24. Hildebrand F, Pape HC, Harwood P, Muller K, Hoevel P, Putz C, et al. Role of adhesion molecule ICAM in the pathogenesis of polymicrobial sepsis. Experim Toxicol Pathol . 2005;56:281-90.

25. Que LG, Kang BH, Huang YC, Piantadosi CA, Chang LY. Antiintercellular adhesion molecule-1 antibody and intercellular adhesion molecule-1 gene deficiency do not prevent pulmonary neutrophil recruitment in polymicrobial sepsis. Shock (Augusta, Ga). 1998;9:304-9.

26. Li N, Yang H, Wang M, Lu S, Zhang Y, Long M. Ligand-specific binding forces of LFA-1 and Mac-1 in neutrophil adhesion and crawling. Mol Biol Cell. 2018;29:408-18.

27. Wiesolek HL, Bui TM, Lee JJ, Dalal P, Finkielsztein A, Batra $\mathrm{A}$, et al. Intercellular adhesion molecule 1 functions as an efferocytosis receptor in inflammatory macrophages. Am J Pathol. 2020;190:874-85.

28. Yang M, Liu J, Piao C, Shao J, Du J. ICAM-1 suppresses tumor metastasis by inhibiting macrophage $\mathrm{M} 2$ polarization through blockade of efferocytosis. Cell Death Dis. 2015;6:e1780.

29. Hubbard AK, Giardina C. Regulation of ICAM-1 expression in mouse macrophages. Inflammation. 2000;24:115-25.

30. Ruetten H, Thiemermann C, Perretti M. Upregulation of ICAM-1 expression on J774.2 macrophages by endotoxin involves activation of NF-kappaB but not protein tyrosine kinase: comparison to induction of iNOS. Mediat Inflamm. 1999;8:77-84.

31. Chen W, Zhong H, Wang X, Pang Q, Zhuang J, Hu J, et al. Mig6 reduces inflammatory mediators production by regulating the activation of EGFR in LPS-induced endotoxemia. J Cell Physiol. 2018;233:6975-83.

32. Hu Y, Lou J, Mao YY, Lai TW, Liu LY, Zhu C, et al. Activation of MTOR in pulmonary epithelium promotes LPS-induced acute lung injury. Autophagy. 2016;12:2286-99.

33. Delano MJ, Ward PA. Sepsis-induced immune dysfunction: can immune therapies reduce mortality? J Clin Investig. 2016;126:23-31.

34. Mayr FB, Yende S, Angus DC. Epidemiology of severe sepsis. Virulence. 2014;5:4-11.

35. van Buul JD, Kanters E, Hordijk PL. Endothelial signaling by Ig-like cell adhesion molecules. Arterioscler Thromb Vasc Biol. 2007;27:1870-6.

36. Welty-Wolf KE, Carraway MS, Huang YC, Simonson SG, Kantrow SP, Kishimoto TK, et al. Antibody to intercellular adhesion molecule
1 (CD54) decreases survival and not lung injury in baboons with sepsis. Am J Respir Crit Care Med. 2001;163:665-73.

37. Ghosh S, Saxena RK. Early effect of Mycobacterium tuberculosis infection on Mac-1 and ICAM-1 expression on mouse peritoneal macrophages. Exp Mol Med. 2004;36:387-95.

38. Gonzalez-Juarrero M, Orme IM. Characterization of murine lung dendritic cells infected with Mycobacterium tuberculosis. Infect Immun. 2001;69:1127-33.

39. Hamerman JA, Aderem A. Functional transitions in macrophages during in vivo infection with Mycobacterium bovis bacillus Calmette-Guerin. J Immunol. 2001;167:2227-33.

40. Lopez Ramirez GM, Rom WN, Ciotoli C, Talbot A, Martiniuk F, Cronstein B, et al. Mycobacterium tuberculosis alters expression of adhesion molecules on monocytic cells. Infect Immun. 1994;62:2515-20.

41. Paulsen K, Tauber S, Dumrese C, Bradacs G, Simmet DM, Golz N, et al. Regulation of ICAM-1 in cells of the monocyte/macrophage system in microgravity. Biomed Res Int. 2015;2015:538786.

42. van Buul JD, Hordijk PL. Endothelial adapter proteins in leukocyte transmigration. Thromb Haemost. 2009;101:649-55.

43. Song R, Ao L, Zhao KS, Zheng D, Venardos N, Fullerton DA, et al. Soluble biglycan induces the production of ICAM-1 and MCP-1 in human aortic valve interstitial cells through TLR2/4 and the ERK1/2 pathway. Inflamm Res. 2014;63:703-10.

44. Song Y, Fullerton DA, Mauchley D, Su X, Ao L, Yang X, et al. Microfilaments facilitate TLR4-mediated ICAM-1 expression in human aortic valve interstitial cells. J Surg Res. 2011;166:52-8.

45. Li J, Jin C, Cleveland JC Jr, Ao L, Xu D, Fullerton DA, et al. Enhanced inflammatory responses to toll-like receptor 2/4 stimulation in type 1 diabetic coronary artery endothelial cells: the effect of insulin. Cardiovasc Diabetol. 2010;9:90.

46. Fan J, Frey RS, Malik AB. TLR4 signaling induces TLR2 expression in endothelial cells via neutrophil NADPH oxidase. J Clin Investig. 2003;112:1234-43.

47. Ode Y, Aziz M, Wang P. CIRP increases ICAM-1(+) phenotype of neutrophils exhibiting elevated iNOS and NETs in sepsis. J Leukoc Biol. 2018;103:693-707.

48. Nakanishi A, Wada Y, Kitagishi Y, Matsuda S. Link between PI3K/ AKT/PTEN pathway and NOX proteinin diseases. Aging Dis. 2014;5:203-11.

49. Hou CH, Lin FL, Tong KB, Hou SM, Liu JF. Transforming growth factor alpha promotes osteosarcoma metastasis by ICAM-1 and PI3K/Akt signaling pathway. Biochem Pharmacol. 2014;89:453-63.

50. Tsoyi K, Jang HJ, Nizamutdinova IT, Park K, Kim YM, Kim HJ, et al. PTEN differentially regulates expressions of ICAM-1 and VCAM-1 through PI3K/Akt/GSK-3beta/GATA-6 signaling pathways in TNF-alpha-activated human endothelial cells. Atherosclerosis. 2010;213:115-21.

51. Muro S, Wiewrodt R, Thomas A, Koniaris L, Albelda SM, Muzykantov VR, et al. A novel endocytic pathway induced by clustering endothelial ICAM-1 or PECAM-1. J Cell Sci. 2003;116:1599-609.

52. Muro S, Garnacho C, Champion JA, Leferovich J, Gajewski C, Schuchman EH, et al. Control of endothelial targeting and intracellular delivery of therapeutic enzymes by modulating the size and shape of ICAM-1-targeted carriers. Mol Ther. 2008;16:1450-8.

53. Serrano D, Bhowmick T, Chadha R, Garnacho C, Muro S. Intercellular adhesion molecule 1 engagement modulates sphingomyelinase and ceramide, supporting uptake of drug carriers by the vascular endothelium. Arterioscler Thromb Vasc Biol. 2012;32:1178-85.

Publisher's Note Springer Nature remains neutral with regard to jurisdictional claims in published maps and institutional affiliations. 